\title{
¿Turismo sostenible o ética del viaje? El valor de las diversidades culturales
}

OLIMPIA NIGLIO

> Kyoto University, Kyoto, Japón. olimpia.niglio@gmail.com

Universidad de Valparaíso

Facultad de Arquitectura

Revista Márgenes

Espacio Arte Sociedad

¿Turismo sostenible o ética del viaje?

El valor de las diversidades culturales

Septiembre 2015 Vol. 12 № 16

Páginas 27 a 33

ISSN elec. 0719-4463

ISSN imp. 0718-4034

Recepción: Agosto 2015

Aceptación: Noviembre 2015

\section{RESUMEN}

El texto analiza la búsqueda de un posible equilibrio entre patrimonio, comunidad, turismo y diálogo intercultural; todos ellos temas de interés general actual y fundamentales para una correcta relación entre los hombres y la sostenibilidad del planeta, pero necesariamente implican también la participación del sistema político y económico. Observando el panorama mundial vemos, sin excepciones, un sistema geopolítico que los expertos definen de resiliencia, es decir de capacidad para reorganizar con éxito la vida en medio de las dificultades y poder reconstruir esta misma vida valorando las oportunidades positivas. Sin embargo, esta condición se puede volver en una forma de deformación negativa sin posibilidad de retorno sino tenemos las herramientas necesarias como para prever y gestionar este proceso de deformación/trasformación. En este artículo se reflexiona sobre estos conceptos partiendo del vínculo existente entre la sostenibilidad y la ética del viaje.

PALABRAS CLAVES

sostenibilidad, viaje, valor ético, patrimonio cultura

\section{Sustainable Tourism or ethical Travel? The value of cultural diversities} ABSTRACT

The paper analyzes the investigation about a possible balance between Heritage, Community, Tourism and Dialogue among culture. Today all these topics are interesting in worldwide and fundamental for a correct relationship among men and for the sustainability of the planet but necessarily all these issues also involve the participation of the political and economic system. Watching the world stage, without exceptions, we see a geopolitical system that the experts call resilience, namely being able to reorganize positively the life compared to the difficulties and be able to reconstruct this life valorizing the positive opportunities. However this situation if will not be prudent it will can turn in a real deformation without return. In this paper we reflect on these issues and on the concept of sustainability and ethics of the trip.

KEYWORDS

sustainability, travel, ethical value, cultural heritage

\section{EL PATRIMONIO CULTURAL}

La Conferencia Mundial de la UNESCO sobre el Patrimonio Cultural, celebrada en México en el año 1982, afirma:

El Patrimonio Cultural de un pueblo comprende las obras de sus artistas, arquitectos, músicos, escritores y sabios, así como las creaciones anónimas, surgidas del alma popular, y el conjunto de valores que dan sentido a la vida, es decir, las obras materiales y no materiales que expresan la creatividad de ese pueblo; la lengua, los ritos, las creencias, los lugares y monumentos históricos, la literatura, las obras de arte y los archivos y bibliotecas (Declaración UNESCO, 1982).
Así entendida la palabra patrimonio incluye al mismo tiempo lo material y lo inmaterial de un pueblo. El concepto de Patrimonio Cultural, en relación a las diversidades sociales y políticas a las que se refiere, tiene un significado mucho más amplio, subjetivo y dinámico y depende principalmente de los valores que las distintas sociedades le atribuyen en cada época histórica. Son estos valores, sobre todos los identitarios, los que determinan los bienes a conservar y proteger para las generaciones futuras. Pero, sobre todo, son estos valores subjetivos, no universales, los que permiten tener una visión más amplia del tema, que la restringida y cerrada que suele darse cuando las referencias son solo las europeas/occidentales (Niglio, 2015:15). 

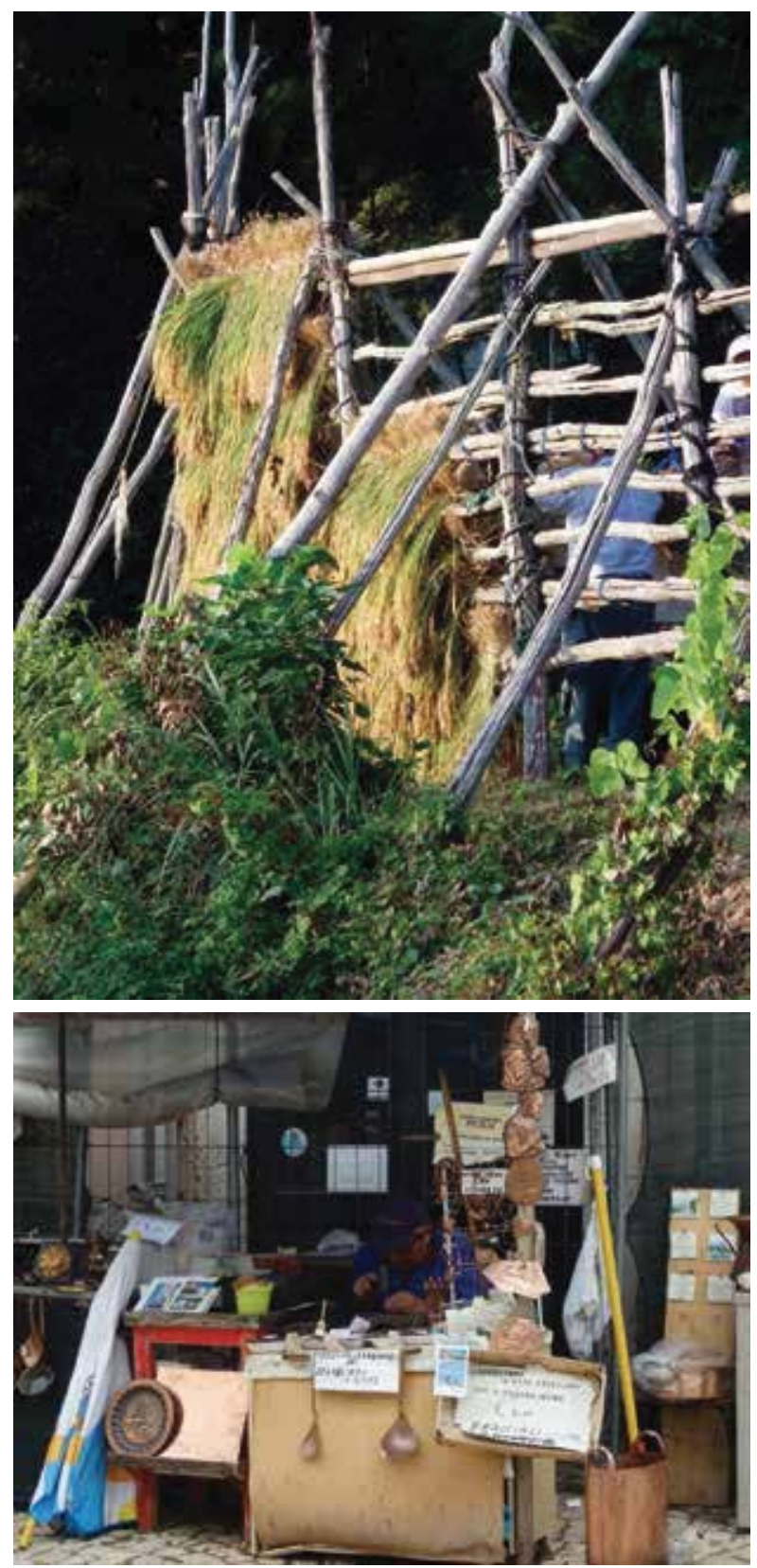

$>$ Figura 1. Japón. Isla de Sado. Antiguo sistema para secar el arroz y hoy los campesinos siguen utilizando esta técnica. Es parte del Patrimonio Cultural local de la isla. Fuente: Olimpia Niglio, 2014.

$>$ Figura 2. Japón. Campo de arroz en Arashiyama, Kyoto. El cultivo se hace con técnicas tradicionales y están consideradas Patrimonio Cultural Intangible del país. Fuente: Olimpia Niglio, 2013

Figura 3. Italia. L'Aquila. Artesano que trabaja con técnicas tradicionales el cobre para hacer artículos domésticos. Fuente: Olimpia Niglio, 2015.

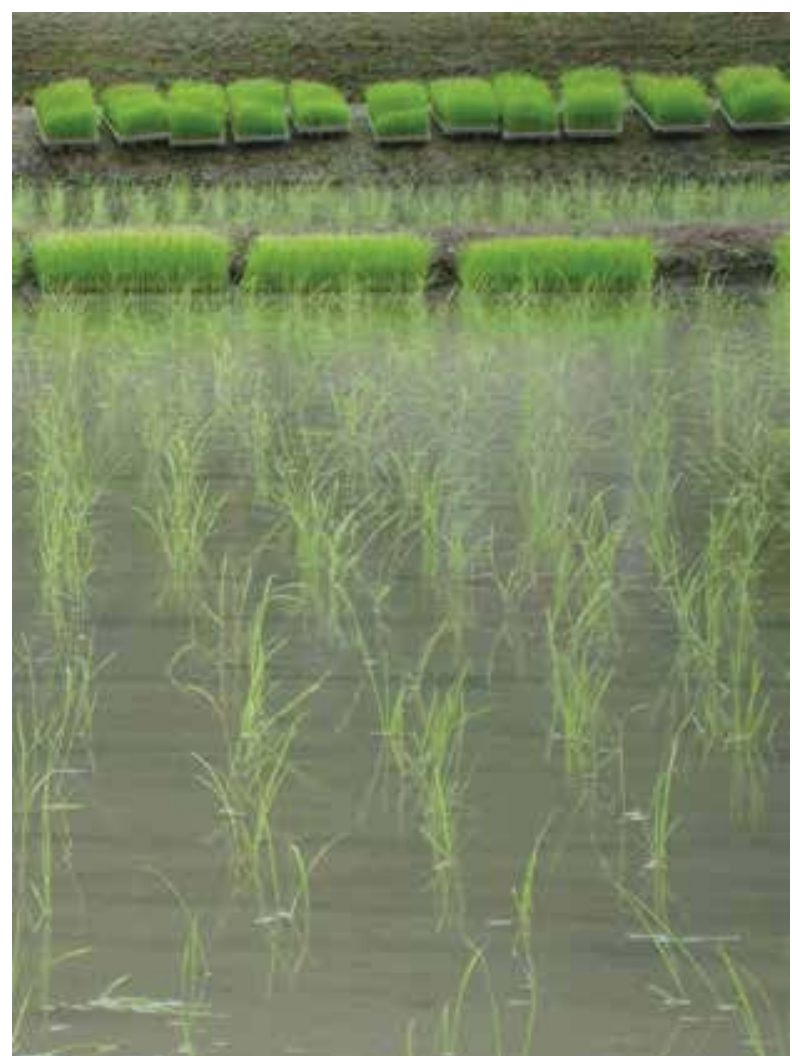

En esta línea, la literatura científica actual propone varios documentos internacionales que analizan el concepto de Patrimonio Cultural y al mismo tiempo consolidan una visión plural del mismo, valorando todos los elementos materiales e inmateriales significativos y testimoniales de las distintas culturas. La bases de estos principios se encontraban ya en la Declaración Universal de los Derechos Humanos (1948) y en el Pacto Internacional de los derechos económicos, sociales y culturales (1966), promoviendo la igualdad de derechos e introduciendo el concepto de personalidad cultural específica de las sociedades, confirmada en la Convención UNESCO sobre la protección y la promoción de las diversidades de las expresiones culturales (2005).

Estos y otros documentos similares, hablan de patrimonio cultural en una dimensión universal, de reconocimientos, de tradiciones y valores, pero a nosotros nos interesa analizar aquí una idea de patrimonio más cercana a las personas que con sus acciones cotidianas lo definen, tal como recuerda Marc Laenen, Director Emérito ICCROM, en su concepto de "sociedad del patrimonio" (Laenen, 2014:31). La sociedad de patrimonio respeta las identidades locales, protege el patrimonio en todos sus aspectos identitarios pero, al mismo tiempo, propone una lectura dinámica del patrimonio que refleje su continua evolución y cambio. Para un correcto análisis de la sociedad de patrimonio es fundamental el aporte de la antropología cultural, la filosofía y el estudio de las distintas religiones, teniendo presente que en la base de todo esto se encuentra la idea de autenticidad del valor cultural - una autenticidad que representa lo propio de la identidad local y toda la herencia de la cultura objeto de estudio. Basándonos solo en los documentos internacionales, vemos que si no se respetan las identidades locales, los valores que cada comunidad reconoce en su herencia cultural y la autenticidad de cada época histórica, no es posible entender cabalmente el concepto de Patrimonio Cultural ni, sobre todo, su visión local y no universal. Solo analizando y respetando el valor local del patrimonio es posible que los viajeros puedan entender 
las distintas visiones del concepto en los diferentes países. Algo básico para desarrollar cualquier proceso de turismo sostenible relacionado con la cultura.

\section{TURISMO Y VIAJE}

A nivel internacional el concepto de Patrimonio Cultural está muy ligado al tema del turismo. Generalizando, el Patrimonio natural y cultural, las culturas y las distintas tradiciones, constituyen uno de los recursos más atractivos del turismo. ¿Sin embargo, aunque parezca obvio, cabe preguntarse qué es realmente el turismo?

Desde una perspectiva operativa, podemos definirlo como un movimiento migratorio temporal que desplaza a una persona o a un grupo de personas desde un punto geográfico a otro caracterizado la temporalidad, pues al final la persona regresa al lugar de partida. La palabra encuentra su fuente en el idioma inglés - tourism-y en el francés -tour-y resulta significativo que el mismo término, turismo, se usa en italiano y en español; pero en todos los casos con esta expresión se entiende una actividad de desplazamiento con un destino y un regreso. Así entendido es, sin duda, una de las actividades más antigua del mundo porque, por distintas razones, todas las comunidades del mundo se han desplazado de un lugar a otro por tiempo determinado. Sin embargo, aunque eran viajes de alguna manera asimilables a los actuales, ¿cabe hablar de turismo en épocas antiguas? Entiendo que no porque el desplazamiento que ha caracterizado la vida de las comunidades antiguas tenía objetivos de conocimiento e intercambio totalmente distintos al significado actual del término. Por eso es fundamental distinguir turismo de viaje.

El viaje implica trasladarse de un lugar a otro, por cualquier medio, con fin de conocer lo auténtico de los lugares y culturas visitados algo implícito en lo que hemos definido como sociedad de patrimonio-y de adaptarse a vivir en ellos dedicando un periodo bastante largo de tiempo a este fin; pensemos en el viaje de Marco Polo en Asia (siglo XIII) o el del escritor Josiah Conder en América Latina (siglo XIX), que no fueron viajes de conquista sino de conocimiento y acercamiento a las culturas de los países anfitriones. Este tipo de viaje se encuentra nuevamente en la Europa del siglo XVIII con la cultura del Grand Tour, donde los ilustrados hacían largos viajes para conocer, quedarse y profundizar en el conocimiento de las culturas de destino.

Por el contrario, el concepto de turismo es algo mucho más cercano al significado moderno de viaje que del comentado desplazamiento activo del viaje, sobre todo en los países con una economía más desarrollada. Solo con la generalización del estado de bienestar de la sociedad burguesa y la consolidación de las clases medias se han puesto las bases para el desarrollo del turismo tal como hoy lo entendemos: una forma de desplazamiento temporal, generalmente muy corto, más privado -incluso egoísta-destinada a favorecer sobre todo el presentismo y no el conocimiento auténtico del lugar visitado. Con el turismo la persona no es un viajero -no es un explorador, un residente temporal, un entusiasta de la cultura local ni un apasionado defensor del nuevo lugar- sino un turista, un consumidor temporal de lugares al que le interesa poder decir que viajó a un determinado país y visitó un monumento famoso; como la Plaza de los Milagros de Pisa en Italia, tomado la consabida foto sujetando la torre inclinada, pero que no sabe que la ciudad tiene un río grande y hermoso, muchas otras iglesias y otros atractivos de interés cultural, pero que no tienen nada a que
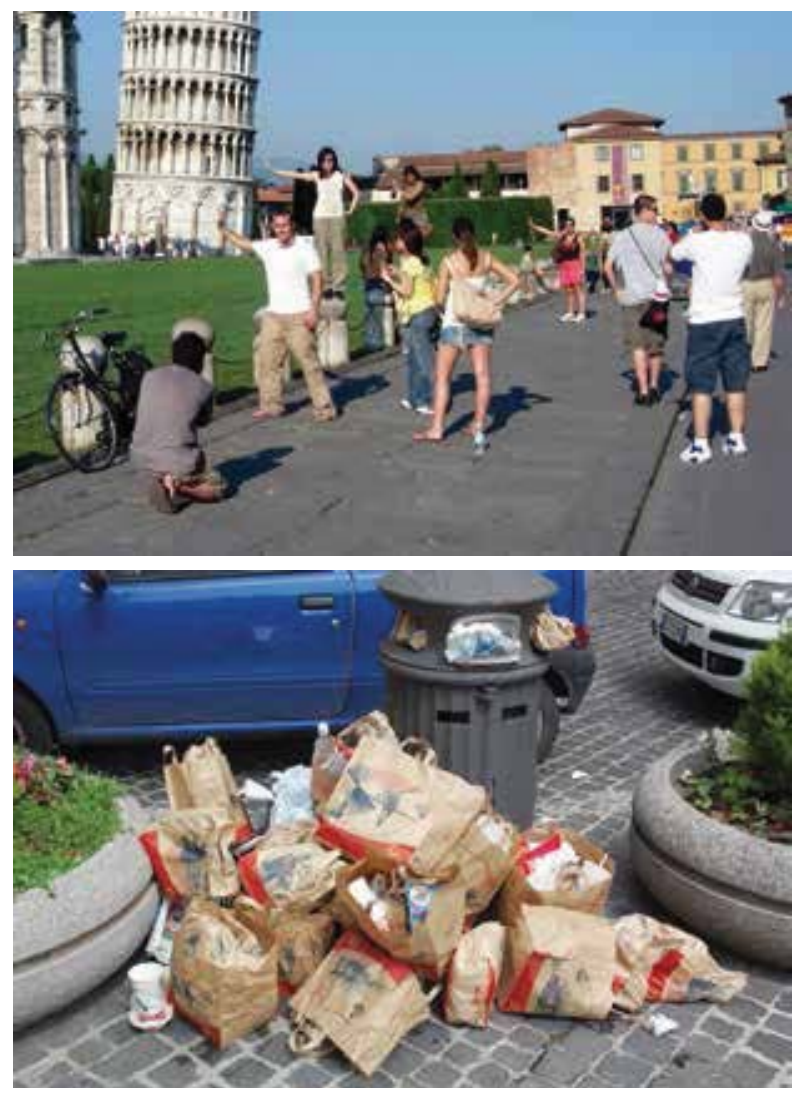

Figura 4. Italia. Pisa. Turistas en Plaza de los Milagros. Fuente: Olimpia Niglio, 2007.

Figura 5. Italia. Roma. Centro Histórico cerca un monumento nacional tras la hora de almuerzo. Fuente: Olimpia Niglio, 2009. 
ver con su cultura especulativa y egoísta. El turista no se relaciona con el lugar donde va, lo consume y parte rápidamente a otro destino sin tener tiempo de entender la cultura local. Todo lo cual se ve hoy facilitado por la tecnología informática, que vende en la misma pantalla las ciudades, los monumentos, los hoteles con piscina, los restaurantes con vistas panorámicas..., todo ello explotado sin tener en cuenta el valor mismo del lugar.

Hoy el interés es especulativo, no interesa fomentar la cultura sino los beneficios económicos que puedan generarse a partir de la cultura. Para potenciar este fenómeno se han ido desarrollando, en los últimos lustros, diversas tipologías de turismo: turismo cultural, turismo ecológico, turismo del deporte y, también, turismo sostenible.

Para nuestro tema, entre estas tipologías merece una atención especial el Turismo sostenible, entendiendo aquí por sostenible la posibilidad de establecer una correcta interacción dinámica y un diálogo positivo entre el turista, el lugar y su Patrimonio Cultural.

\section{¿TURISMO SOSTENIBLE O VIAJE ÉTICO?}

Hablar de sostenibilidad del turismo significa acercar más esta expresión a cinco principales áreas de evaluación:

Redefinir el concepto de viajero, como posible residente temporal de corto plazo y con distintos intereses que dialogan y no afectan el lugar que lo alberga. Todo eso es posible fortaleciendo el conocimiento que ayuda a respectar el lugar y la comunidad local.

Favorecer el conocimiento de la cultura y las características propias del lugar visitado con el fin de establecer un diálogo entre la comunidad local y los viajeros.

Profundizar en una educación del conocimiento con la colaboración de las comunidades escuelas locales, porque este tipo de educación permite de acercar más los jóvenes a su patrimonio cultural, apreciarlo, protegerlo y, al mismo tiempo, adquirir herramientas para respetar también el patrimonio cultural de otras culturas. Esto implica diálogo entre culturas.

Potenciar una comunicación cultural que favorezca la evolución dinámica de la cultura y contribuya a una correcta difusión del conocimiento.

Promover acciones de gestión cultural donde el interés sea realmente el conocimiento del lugar y su descubrimiento para fortalecer las oportunidades de diálogo y encuentro.

Cumplir con estos objetivos puede tener un gran potencial pues permitiría pasar del concepto de turismo sostenible al de viaje ético, en un sentido más contemporáneo del mismo, al estar orientado al conocimiento y el encuentro entre distintas culturas. El viaje ético significa: establecer una estrecha relación entre el viajero y el residente del lugar visitado, acercar al viajero a las necesidades de una comunidad que garantice la hospitalidad con el fin de crear un correcto equilibrio entre el uso del lugar y el conocimiento de las riquezas de los valores del mismo. Todo esto, también, con la finalidad de ofrecer al viajero un conocimiento adecuado de un patrimonio que no es fácil entender sin esta relación positiva con la comunidad local.

La conciencia del lugar, de su paisaje cultural y de los valores de la comunidad local puede ayudar (mucho) a producir un consumo sostenible y a lograr una protección adecuada de los valores locales y, por lo tanto, a favorecer un diálogo intercultural entre viaje- ros y comunidades anfitrionas. Aspecto hoy muy importante para un correcto y tolerante diálogo entre las diversidades culturales (Rypkema, 2012:131).

\section{VIAJE ÉTICO Y DIVERSIDADES CULTURALES}

El concepto común de turismo encarna un fenómeno global que ha transformado, en menos de un siglo, el concepto de viaje -con todos sus contenidos asociados de descubrimiento y de conocimiento- en algo orientado sobre todo a los servicios de consumo.

Gracias a la evolución tecnológica hoy es más fácil moverse de un país a otro, de comunicarse con pueblos de otros idiomas, con creencias religiosas, ideologías culturales y estructuras políticas distintas. Sin embargo, no siempre las nuevas tecnologías están favoreciendo la comprensión de las culturas y el diálogo intercultural. Es más, generalmente prevalece una forma de ignorancia conjunta a la tolerancia entre los visitantes y la comunidad residente que, en muchos casos, tiene solo el interés de disfrutar la oportunidad del fenómeno turístico sin aprovecharlo para un mejoramiento cultural de este encuentro entre visitantes y residentes locales.

Por ello nos parece muy interesante empezar a trasformar -como hemos apuntado- el concepto de turismo en viaje ético, con el fin de fomentar el conocimiento y encuentro entre distintas culturas. A todo eso se une la conservación y la valorización del Patrimonio; sin olvidar que las mismas diversidades culturales pueden considerarse valores del lugar que permiten fortalecer acciones de protección e intercambio. El encuentro entre culturas no significa globalización, es exactamente el contrario. Es el respecto de las distintas tradiciones locales en un proceso de conocimiento amplio, dinámico y activo. Sólo un diálogo que tenga como base estos principios éticos puede contribuir a intercambiar valores, a no destruir y a promover la paz. En un momento histórico muy complejo como el que estamos viviendo en todo el mundo, y por distintas razones sociales y políticas, es fundamental insistir en el valor ético del viaje y de las relaciones multiculturales que puede promover.

\section{VIAJE ÉTICO Y PATRIMONIO}

En este diálogo multicultural es importante afrontar el encuentro entre viaje y patrimonio y sobre todo analizar el tema con referencia a varios puntos críticos propios del sistema que impone hoy el desplazamiento de un punto a otro del mundo:

Las modalidades de la organización del viaje (en particular la política de inmigración) a nivel mundial, respecto a la finalidad misma del viaje (vacaciones, estudio, trabajo, trasferencia, refugiados, etc.).

La sostenibilidad económica del Patrimonio Cultural del país anfitrión garantizada por una política de participación e involucramiento de los viajeros en la tutela de la cultura local, entendida como patrimonio de todos.

El valor del viaje como oportunidad de conocimiento de las distintas culturas.

Los efectos del proceso de desplazamiento de las personas y su gestión política, cultural y económica.

Una correcta relación entre los servicios ofrecidos, gastos y rentas respecto del Patrimonio Cultural local. En particular, parte de las rentas deben ser destinadas a la protección y mantenimiento del Patrimonio Cultural; así el propio viajero sabe que con su viaje contribuye a salvaguardar el patrimonio mundial. Todo eso significa 

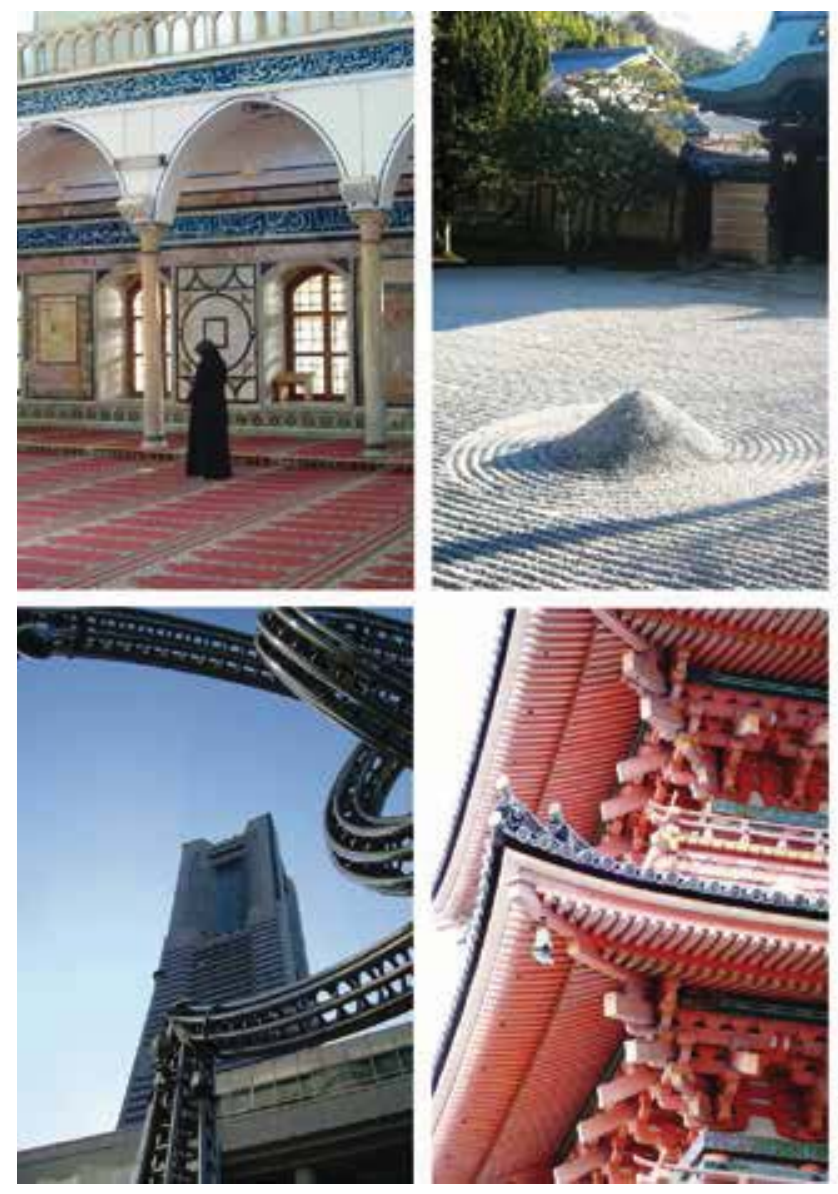
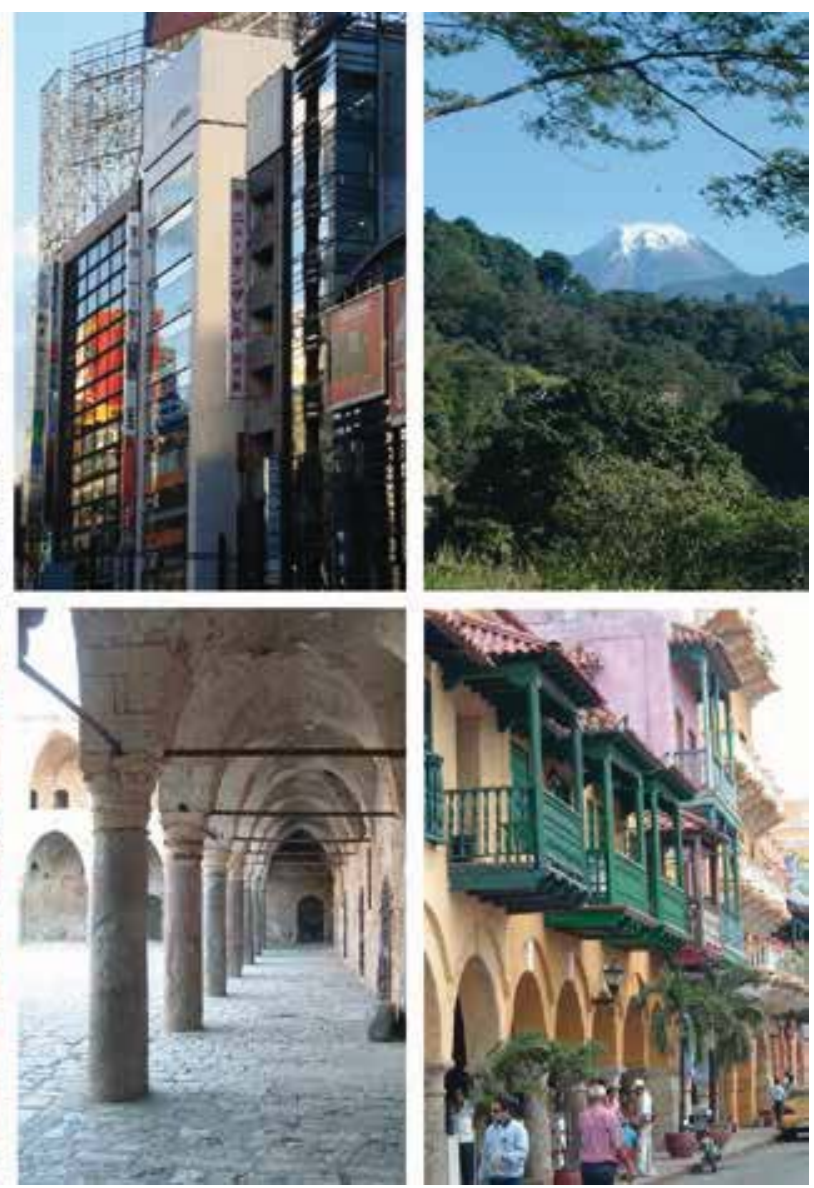

sostenibilidad local promovida gracias: 1. a la autoconciencia y respeto de las comunidades de cada país por el propio Patrimonio tangible e intangible y por su valor cultural, al mismo tiempo histórico y contemporáneo; 2 . a la comunicación entre las distintas culturas y la valorización de sus patrimonios interpretado en su entorno.

Esta sostenibilidad local así desarrollada puede favorecer una correcta fruición del patrimonio mundial; dejar a los viajeros claves de lectura adecuada para conocer los significados y personalidades de los distintos territorios; poner a la comunidad local en diálogo con los viajeros y aprovechar este encuentro para la valorización y gestión del patrimonio; algo que no sólo incumbe a la comunidad local sino a todo el mundo. Es esta afiliación la que hoy, más de ayer, es fundamental para preservar y respetar las distintas culturas. Obviamente el objetivo fundamental de esta sostenibilidad local, favorecida gracias al viaje ético, es crear una profunda armonía multicultural y el reconocimiento mutuo entre las comunidades, preservando al mismo tiempo los derechos humanos y los múltiples valores del planeta. Sobre esto es interesante leer la Carta internacional sobre turismo cultural, adoptada por ICOMOS en la $12^{\text {a }}$ Asamblea General en México, en octubre 1999, que introduce muchos temas fundamentales para reflexionar sobre la sostenibilidad de la actividad turística y analizar la interacción activa y dinámica entre Viaje / Turismo y Patrimonio Cultural, las diversidades y las culturas vivas, las tradiciones, la planificación de políticas concretas de desarrollo y los programas de interpretación y difusión del valor del patrimonio. Todos estos temas constituyen factores esenciales para construir una sostenibilidad local donde las comunidades anfitrionas y los viajeros puedan involucrarse en la planificación de la conservación del patrimonio y en la planificación misma del viaje (Carta Turismo Cultural ICOMOS, 1999). $>$ Figura 6. La multiculturalidad del patrimonio tangible. Fuente: Olimpia
Niglio, 2011. 

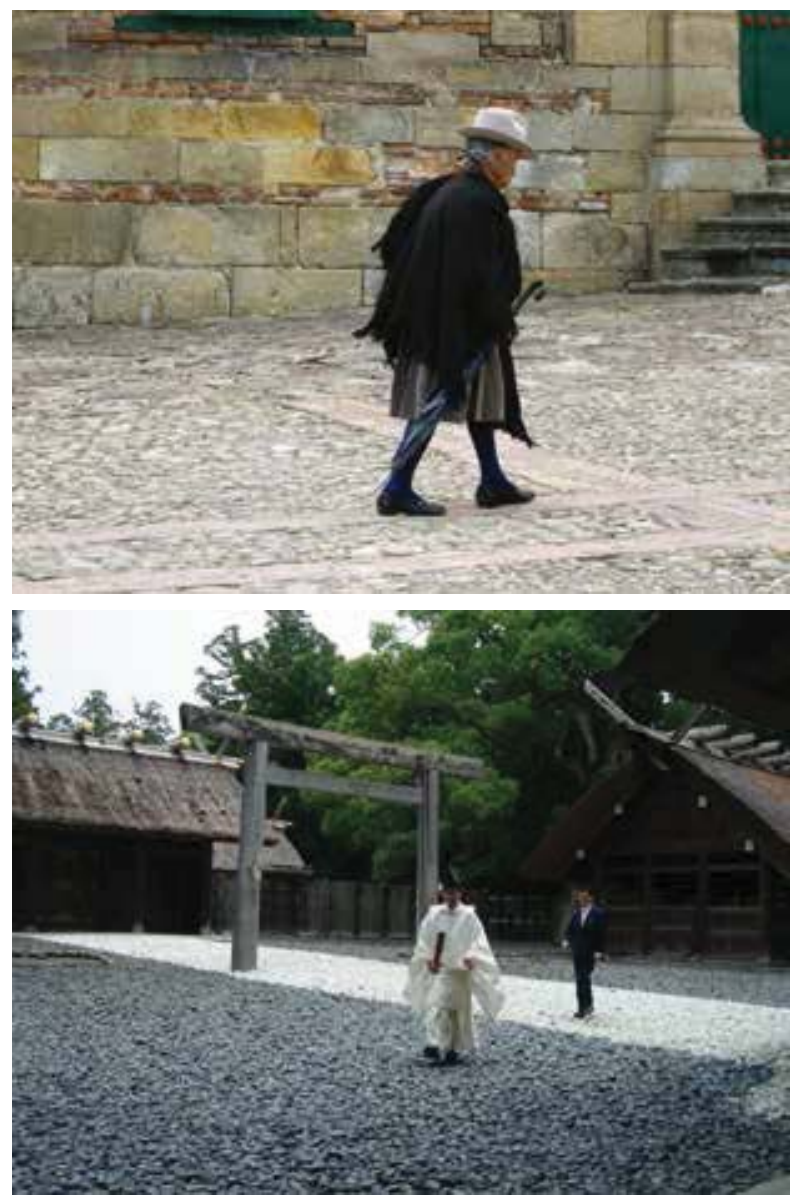

Figura 7. Colombia. Monguí. Cultura local y valores éticos. Fuente: Olimpia Niglio 2014.

$>$ Figura 8. Japón. Ise, Santuario Geku. Ceremonia scintoista. Fuente: Olimpia Niglio, 2013.

\section{LOS OBJETIVOS DE LA SOSTENIBILIDAD LOCAL}

En el último informe de la Comunidad Europea (Getting cultural heritage to work for Europe, 2015:13) se lee:

In the last fifteen years many local and regional entrepreneurs have developed successful strategies in sustainable tourism that combine local and regional strategies on biodiversity with the protection of cultural heritage and the production of high quality regional products.

Portugal e Italia han sido dos países europeos muy sensibles a esta política de diálogo entre Patrimonio Cultural y desarrollo regional y han promovido proyectos muy interesantes. En general, estos proyectos han permitido lograr no sólo beneficios económicos sino también beneficios culturales, donde el propio ciudadano participa en la promoción de su territorio con conciencia y con el fin de desarrollar la sostenibilidad local.

The availability of cultural heritage and services is not only important for its measurable economic benefits. It also enriches the quality of life for European citizens and contributes to their wellbeing, sense of history, identity and belonging. Such social benefits are beyond what can be measured in terms of pure income statistics and have been long recognized. As early as the $14^{\text {th }}$ century, the Statutes of independent Italian municipalities attributed to cultural heritage foreign visitors' happiness and residents' honour and prosperity, based on beauty, embellishment (decorum), dignity, public pride and public good (publica utilitas) (Getting cultural heritage to work for Europe, 2015:7).

En los países de Extremo Oriente y en particular en Japón, este concepto de sostenibilidad local es parte de la cultura común; se aprende en casa, en las escuelas, desde pequeño, creando una sensibilidad frente al patrimonio que constituye un bien enorme que la gente se lleva de por vida y fomenta el respeto por su propia cultura, las otras civilizaciones y, sobre todo, ayuda a planear el futuro del propio Patrimonio Cultural, tangible e intangible. De este enfoque cultural hay mucho que aprender para definir con mayor claridad los objetivos de la sostenibilidad.

La agencia internacional United Nations World Tourism Organization (UNWTO), lleva varios años trabajando sobre la importancia de la planificación de una política dirigida a la sostenibilidad de los territorios y al diálogo entre turismo, viajeros, ciudadanos y patrimonio cultural local y ha planteado la necesidad de reflexionar sobre estos 12 presupuestos que son fundamentales para lograr la sostenibilidad local (UNWTO, 2004:53):

- Viabilidad económica

- Prosperidad local

- Calidad de empleo

- Equidad social

- Satisfacción del visitante

- Control local

- Bienestar de la comunidad

- Riqueza cultural

- Integridad Física

- Diversidad biológica

- Eficacia en el uso de los recursos

- Pureza ambiental 
Un análisis puntual de estos objetivos nos permite evaluar y realizar correctamente la integración entre viajero y patrimonio cultural, entre políticas de desarrollo nacionales e internacionales; así como proponer la relación Turismo / Viaje como principal fuente de financiación para la conservación del patrimonio cultural y fuente de desarrollo para las comunidades locales; de reflejar sobre los distintos valores del patrimonio cultural y, gracias a esto, de valorar el diálogo intercultural, así como la posibilidad de promover una política de gestión medioambiental, territorial y cultural orientada a la preservación de la integridad y autenticidad del patrimonio cultural local y de su historia. (Burke, 2003:76). Todo eso es un proyecto de sostenibilidad local cuyo fin es respetar las diversidades culturales y promover sus valores.

\section{OBSERVACIONES FINALES PARA INICIAR EL VIAJE ÉTICO}

Consideramos que es posible conseguir una sostenibilidad local si en la base de cualquier proyecto turístico existe una ética del conocimiento y una ética de la hospitalidad. Sin cultura del conocimiento y sin cultura de la hospitalidad es muy difícil respetar los lugares, las comunidades y las distintas culturas con su Patrimonio Cultural. Por eso hemos introducido el concepto de viaje ético que permite relacionar la comunidad local y los viajeros en una relación biunívoca. Sólo el conocimiento y comparación entre distintas culturas nos permitirá resolver muchos temas propios del tercer milenio así como la igualdad social, el respeto de los derechos humanos, la paz y el desplazamiento de muchas comunidades por problemas de inseguridad social, guerras y desigualdades.

El viaje ético nos permitirá de leer las realidades con ojos más atentos, menos egoístas y sobre todo más relacionados con las necesidades de un mundo que hoy -gracias también a las tecnologías- cambia muy rápidamente. Este tipo de viaje podemos compararlo a la visión de la época romántica, desde el siglo XVIII hasta el siglo XIX, que había renunciado a la concepción del viaje descriptivo y objetivo para pasar a valorar las sensaciones y las vivencias del viajero y donde el imaginario y la atracción por lo desconocido tenía un valor divino (Flaubert, 1993). Es esta subjetividad asociada con la tolerancia y con el deseo de conocer realidades distintas la que favorecerá el principio de nuestro viaje ético para el respeto de la humanidad.

\section{BIBLIOGRAFÍA}

AA. VV. (2015), Getting cultural heritage to work for Europe. Report of the Horizon 2020. Expert Group on Cultural Heritage, Brussels: European Commission.

BURKE, P. (2003), "Historia social del conocimiento: de Gutenberg a Diderot”, Barcelona: Paidós.

FLAUBERT, G. (1993) “Viaje a Oriente”, Madrid: Cátedra.

LAENEN, M. (2014), From heritage conservation to its social fruition for society and humanity: the interpretation and presentation of the cultural biography of living environments/ cultural landscapes: travel for intercultural dialogue. En AA.VV., "Travel for Dialogue. With heritage for sustainable development”, pp. 93-98, Florence: Romualdo Del Bianco Foundation.

NIGLIO, O. (2015), "El valor del patrimonio cultural entre extremo Oriente y extremo Occidente", Roma: Aracne.
RYPKEMA, D. (2012), Heritage Conservation and Property Values, en: LICCIARDI, G.; AMIRTAHMASEBI, R., "The Economics of Uniqueness, Investing in Historical City Cores and Cultural Heritage Assets for Sustainable Development", The World Bank, pp. 107-142.

UNWTO (2004), Tourism Congestion Management at Natural and Cultural Sites, Madrid.

\section{DOCUMENTOS INTERNACIONALES}

Declaration on Cultural Policies World Conference on Cultural Policies, Mexico City, 26 July - 6 August 1982, UNESCO. http://portal.unesco.org/culture/en/ files/12762/11295421661mexico_en.pdf/mexico_ en.pdf, consulta: 29.06.2015.

Carta internacional sobre turismo cultural. La Gestión del Turismo con Patrimonio Significativo, ICOMOS, $12^{\mathrm{a}}$ Asamblea General, México, 1999, http://www.international.icomos. org/charters/ tourism_sp.pdf, consulta: 29.06.2015. 